\title{
Comparison of Highly-Fluorinated Chloroformates as Direct Aqueous Sample Derivatizing Agents for Hydrophilic Analytes and Drinking-Water Disinfection By-Products
}

\author{
Marco Vincenti, Stefano Biazzi, Nicoletta Ghiglione, \\ and Maria Carmen Valsania \\ Dipartimento di Chimica Analitica, Università di Torino, Torino, Italy
}

\author{
Susan D. Richardson \\ National Exposure Research Laboratory, United States Environmental Protection Agency, Athens, Georgia, \\ USA
}

Four highly-fluorinated alkyl and aryl chloroformates, including 2,2,3,3,4,4,5,5-octafluoro-1pentyl chloroformate (OFPCF), 2,3,4,5,6-pentafluorobenzyl chloroformate (PFBCF), 3,3,4,4,5,5,6,6,7,7,8,8,8-tridecafluoro-1-octyl chloroformate (TDFOCF), and 2-(2,3,4,5,6pentafluorophenoxy)-ethyl chloroformate (PFPECF), were synthesized and tested as reagents for the direct water derivatization of polar and hydrophilic analytes. The goal of this research was to develop an optimal derivatizing agent to aid in the identification of highly polar ozonation drinking water disinfection by-products (DBPs) that are believed to be missed with current analytical procedures. The chemical properties (reactivity, selectivity, derivatization products, and their chromatographic and spectral features) for the four chloroformates were investigated using a set of highly polar standard analytes, including malic and tartaric acids, hydroxylamine, valine, 2-aminoethanol, resorcinol, 1,3,5-trihydroxybenzene, and 2,4-dihydroxybenzoic acid. Upon derivatization, the analytes were extracted from the aqueous solvent and analyzed by gas chromatography (GC)-mass spectrometry (MS) in the electron capture negative ionization (ECNI) mode. Positive chemical ionization (PCI)-MS was used for confirmation of molecular ions that were weak or absent in ECNI mass spectra. Of the four derivatizing reagents tested, OFPCF showed the best performance, with good reaction efficiency, good chromatographic and spectroscopic properties, low detection limits (10-100 $\mathrm{fmol}$ ), and a linear response more than two orders of magnitude. Further, the entire procedure from raw aqueous sample to ready-to-inject hexane solutions of the derivatives requires less than $10 \mathrm{~min}$. PFBCF showed ideal applicability for derivatizing aminoalcohols and aminoacids. The two chloroformates with the highest intrinsic stability (TDFOCF and PFPECF) failed to derivatize some of the analytes. Finally, the OFPCF derivatizing agent was tested with simulated ozonated drinking water (aqueous fulvic acid treated with ozone), and three highly polar reaction by-products were determined. (J Am Soc Mass Spectrom 2005, 16, 803-813) () 2005 American Society for Mass Spectrometry

$\mathrm{B}$ oth environmental and clinical analyses commonly use mass spectrometry (MS) to detect organic compounds at trace level in aqueous matrices. However, the identification and detection of the smallest and most hydrophilic organic analytes presents a difficult task, as these substances cannot be extracted into an organic solvent nor concentrated [1]. Moreover, their direct detection in the original matrix

Published online March 30, 2005

Address reprint requests to Dr. M. Vincenti, Dipartimento di Chimica Analitica, Università di Torino, Via Pietro Giuria 5, 10125 Torino, Italy. E-mail: marco.vincenti@unito.it by liquid chromatography (LC)-MS can be difficult, because of the high background chemical noise in the low $\mathrm{m} / \mathrm{z}$ region of the spectrum.

Small, highly hydrophilic organic disinfection byproducts (DBPs) are produced in drinking water treatment by the reaction of disinfectants with the natural organic matter (e.g., humic and fulvic acids) [2-4] and anthropogenic substances released from wastewaters. To date, more than 500 DBPs have been identified [2, $5-8$ ], yet approximately $63 \%$ of the assimilable organic carbon (AOC) produced in ozonated drinking water is not accounted for [1]. Particularly for ozone, it is believed that many of DBPs are highly hydrophilic and 
are not being detected with current analytical techniques. DBPs of this kind are likely to be polyacids, hydroxyacids, ketoacids, glyoxals, hydroxylamines, aminoacids, aminoalcohols, and glycols [9].

Direct sample derivatization in water reverses the more typical sequence of derivatization after extraction, by converting the analyte's polar groups to more hydrophobic ones, enabling their extraction into organic solvents. However, most derivatizing agents undergo immediate hydrolysis as soon as they are introduced into water. Alkyl chloroformates are among the few derivatizing agents that tolerate water [10], and $n$-hexyl chloroformate has been successfully demonstrated in previous reports [11-15].

Despite the high derivatization efficiency of $n$-hexyl chloroformate with carboxylic, hydroxylic, and aminic compounds, polysubstituted products obtained from tri- and tetra-functional analytes (e.g., tartaric acid, dihydroxybenzoic acid) are not volatile and cannot be easily eluted from a GC column. To overcome this drawback and improve sensitivity, we set out to synthesize highly-fluorinated alkyl and aryl chloroformates [16, 17] for the derivatization and detection (by ECNI-MS) of a large variety of highly polar compounds.

Trifluoroethyl chloroformate [18] and pentafluorobenzyl chloroformate (PFBCF) $[19,20]$ were previously synthesized and used by other groups for the direct derivatization of aminoacids in human plasma and whole blood, followed by ECNI-MS detection [21]. We recently developed a rapid, safe, and efficient procedure to synthesize highly-fluorinated chloroformates on a small scale needed for analytical applications [17]. In the present study, we compared the analytical performance of four highlyfluorinated chloroformate derivatizing agents: (1) PFBCF; (2) 2,2,3,3,4,4,5,5-octafluoropentyl chloroformate (OFPCF); (3) 3,3,4,4,5,5,6,6,7,7,8,8,8-tridecafluorooctyl chloroformate (TDFOCF); and (4) 2-(2,3,4,5,6-pentafluorophenoxy)ethyl chloroformate (PFBECF). The structures of these reagents are reported in Scheme 1. OFPCF and PFBCF had been successfully used in the past $[16,19,20]$, while TDFOCF and PFPECF were selected in the present study since the longer hydrocarbon spacer located between their reactive chloroformate group and the fluorinated moiety
(Scheme 1) was expected to increase their intrinsic stability. Finally, the applicability of one of these reagents (OFPCF) was demonstrated by identifying ozone DBPs in simulated drinking water.

\section{Experimental}

\section{Derivatization Procedure}

Highly-fluorinated chloroformates were synthesized from the corresponding alcohols (all commercially available from Aldrich, Milan, Italy) using either acetone or $n$-pentane as the reaction solvent [17]. Acetone was preferred for most analytical applications. All details on the specific syntheses (reaction yields, purities, etc.) are provided elsewhere [17]. The resulting chloroformate solutions proved stable for at least two months, if properly sealed.

Aqueous samples ( $2 \mathrm{~mL}$ for all the experiments of the present work) were basified with $100 \mu \mathrm{L}$ of $3 \mathrm{M} \mathrm{NaOH}$. Then, $150 \mu \mathrm{L}$ of chloroformate solution was added ( $2 \mu \mathrm{mol}$ of chloroformate in acetone), while keeping the reaction tube under ultrasonic mixing at optimized power and distance from the emitting tip. Immediately, $3 \mu \mathrm{L}$ of a saturated $(400 \mathrm{~g} / \mathrm{L})$ dicyclohexylcarbodiimide (DCC) solution in pyridine was added. The reaction was allowed to proceed for 5-10 min under sonication. The reaction products were extracted in $n$-hexane (600 $\mu \mathrm{L}$ ) over $1 \mathrm{~min}$. The organic layer was separated and analyzed by GC-MS. All the derivatization products proved stable for at least several hours. WARNING: Ears should be protected with appropriate earplugs during sonication. The reported experimental conditions result from extensive optimization of the following parameters: amount of derivatizing agent, amount of catalyst, sonication time, and amount of base added.

Pyridine is used as a catalyst, while DCC is a coupling agent that increases derivatization yields and repeatability. Crude chloroformate solutions in acetone, used in the derivatization, still contain small amounts of $\mathrm{HCl}$ and phosgene that are converted into inert $\mathrm{NaCl}$ and $\mathrm{NaCO}_{3}$, upon $\mathrm{NaOH}$ addition.

With respect to the derivatization procedure previ-

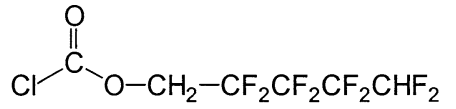

OFPCF

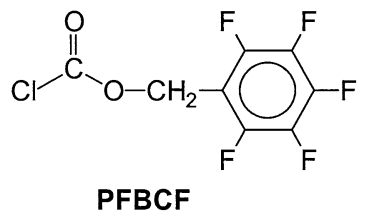

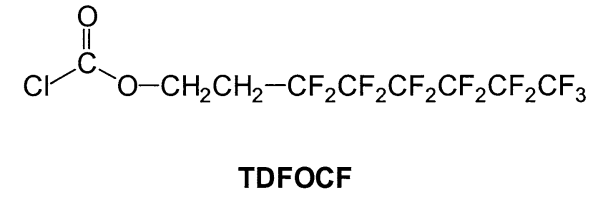

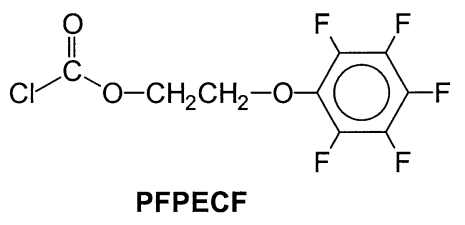


ously published [16], the reaction media contains about $7 \%$ of organic solvent, either pentane or acetone. While the latter has virtually no impact on the derivatization efficiency, the presence of $n$-pentane proved detrimental for the most polar analytes (i.e., hydroxylamine, tartaric acid, 2-aminoethanol), in terms of detection limits (up to 15 times lower) and reproducibility (the standard deviation of repeated derivatizations could exceed $40 \%$ ). These effects are attributed to the presence of two phases, preventing an effective interaction between analytes and the derivatizing agent.

\section{Instrumentation and Analysis}

A Branson Sonifier II W-450 (Danbury, CT) sonicator, with variable emission power, was used to enable the derivatizations with ultrasonic mixing. To assure optimal and reproducible mixing, a reaction bath was built from high-vacuum spare parts. However, similar results could be obtained using an ordinary water bath, but mechanical stirring should be avoided. The derivatizations were performed in a glass test tube maintained in a fixed vertical position by a rubber o-ring and a guide in the stainless steel reversed-T structure of the bath (Figure 1 ). ${ }^{\circ}$ The $^{\circ}$ sonifier ${ }^{\circ}$ tip $^{\circ}$ was $^{\circ}$ screwed $^{\circ}$ horizontally into the water bath from an opening on the lateral closure disk so as to produce maximum emission power over a 1 to $2 \mathrm{~cm}^{2}$ area in the center of the bath. The ultrasonic emission contacted the reaction vessel perpendicularly from a fixed $1 \mathrm{~cm}$ distance at which the radiation was homogeneously distributed in the reaction volume. The emission power was optimized by a series of derivatizations in which the reaction yields were evaluated as a function of the sonication power. The mixing efficiency proved to increase with the emission power up to $160 \mathrm{~W}$ and then leveled off. This corresponds to an effective power of about $90 \mathrm{~W}$ released to the medium.

A benchtop Perkin Elmer TurboMass (Norwalk, CT) spectrometer equipped with an AutoSystem XL gas chromatograph was utilized for most analyses. The quadrupole analyzer had a $\mathrm{m} / \mathrm{z}$ range of 1200 . A chemical ionization source was used to acquire both PCI and

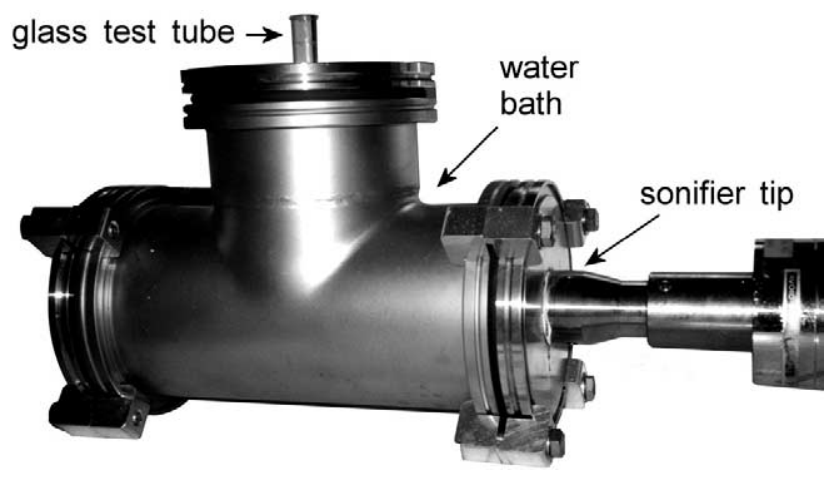

Figure 1. Derivatization reaction bath, operating under ultrasonic mixing.
ECNI mass spectra. Isobutane was employed as the reagent gas for both positive and negative ion experiments, at a pressure of $50 \mathrm{~Pa}$. The ion source was maintained at the lowest temperature $\left(150^{\circ} \mathrm{C}\right)$, compatible with prevention of analyte condensation.

A DB-5MS (5\% diphenyl dimethyl siloxane) capillary column (30 m long, $0.25 \mathrm{~mm}$ i.d., $0.25 \mu \mathrm{m}$ film thickness, Agilent, Folsom, CA) was utilized. The samples were injected by an AutoSystem XL autosampler in the splitless mode at a temperature of $270{ }^{\circ} \mathrm{C}$. The carrier gas (helium) was maintained constant at $1 \mathrm{~mL} / \mathrm{min}$. The oven temperature was programmed as follows: isothermal at $50{ }^{\circ} \mathrm{C}$ for $3 \mathrm{~min}$, from 50 to $300{ }^{\circ} \mathrm{C}$ at $15^{\circ} \mathrm{C} / \mathrm{min}$, isothermal at $300{ }^{\circ} \mathrm{C}$ for $10 \mathrm{~min}$. The transfer line was maintained at $250^{\circ} \mathrm{C}$.

For occasional confirmation and mass assignment in the range above the quadrupole limit, a Finnigan-MAT 95Q (Bremen, Germany) magnetic sector mass spectrometer $(\mathrm{m} / \mathrm{z}$ limit of 3500$)$ was utilized under similar conditions, ${ }^{\circ}$ as $^{\circ} \operatorname{described~}^{\circ}$ in $^{\circ}$ detail $^{\circ}$ elsewhere ${ }^{\circ}[16]^{\circ}$ Scan ranges varied depending on the derivatives to be determined; typical ranges were $m / z 250-920$ or 300-1300, scanned at a rate of $1 \mathrm{~s} /$ decade.

\section{Ozonation of Fulvic Acids}

Laboratory-scale batch ozonations were carried out at $\mathrm{pH} 7$ by the addition of ozone (from a $30 \mathrm{mg} / \mathrm{L}$ freshly prepared, stock solution of ozone in water, at a dose of 2:1 ozone to dissolved organic carbon) to solutions of Suwannee River fulvic acid (SRFA, International Humic Substances Society, St. Paul, MN) in purified water (5 mg/L) containing $1 \mathrm{mM}$ sodium bicarbonate buffer. SRFA was used as a natural organic matter constituent because it is a well-characterized organic matter, and fulvic acid has been previously shown to be a major precursor ${ }^{\circ}$ to $^{\circ}$ the ${ }^{\circ}$ formation ${ }^{\circ}$ of $^{\circ} \mathrm{DBPs}^{\circ}[2] .{ }^{\circ} \mathrm{Ozone}^{\circ}$ stock solutions were prepared by sparging ozone from an Orec ozone generator (Osmonics, Phoenix, AZ) into purified water maintained close to $0{ }^{\circ} \mathrm{C}$. Ozone concentrations were determined by measuring the UV absorbance (at $254 \mathrm{~nm}$ ) of the stock solution and dividing by the molar absorptivity of ozone $\left(2950 \mathrm{M}^{-1} \mathrm{~cm}^{-1}\right)$ and the length ${ }^{\circ}$ of $^{\circ}$ the $^{\circ}$ light $^{\circ}$ path $^{\circ}$ through $^{\circ}$ the $^{\circ}$ sample $^{\circ}[22] .{ }^{\circ} \mathrm{A}$ purified water blank (containing fulvic acid and buffer, but not ozonated) was also derivatized and analyzed as a control. Samples were collected after $3 \mathrm{~h}$ of reaction with ozone.

\section{Results and Discussion}

Reaction with chloroformates converts carboxylic acids into the corresponding esters, whereas alcohols and phenols form carbonates, and amines form carbamates $\left(\text { Scheme }^{\circ} 2\right)^{\circ}\left[10,{ }^{\circ} 14\right] .{ }^{\circ}$ For $^{\circ} \operatorname{primary}^{\circ} \operatorname{amines}^{\circ}\left(\mathrm{R}_{2}=\mathrm{H}\right.$ in Scheme 2), one or both aminic hydrogens undergo substitution, depending on the type of chloroformate used $^{\circ}$ and $^{\circ}$ the $^{\circ}$ analyte $^{\circ}$ reactivity $^{\circ}\left[14,{ }^{\circ} 16\right] .^{\circ}$ Reactivity differences among the four chloroformates were tested 

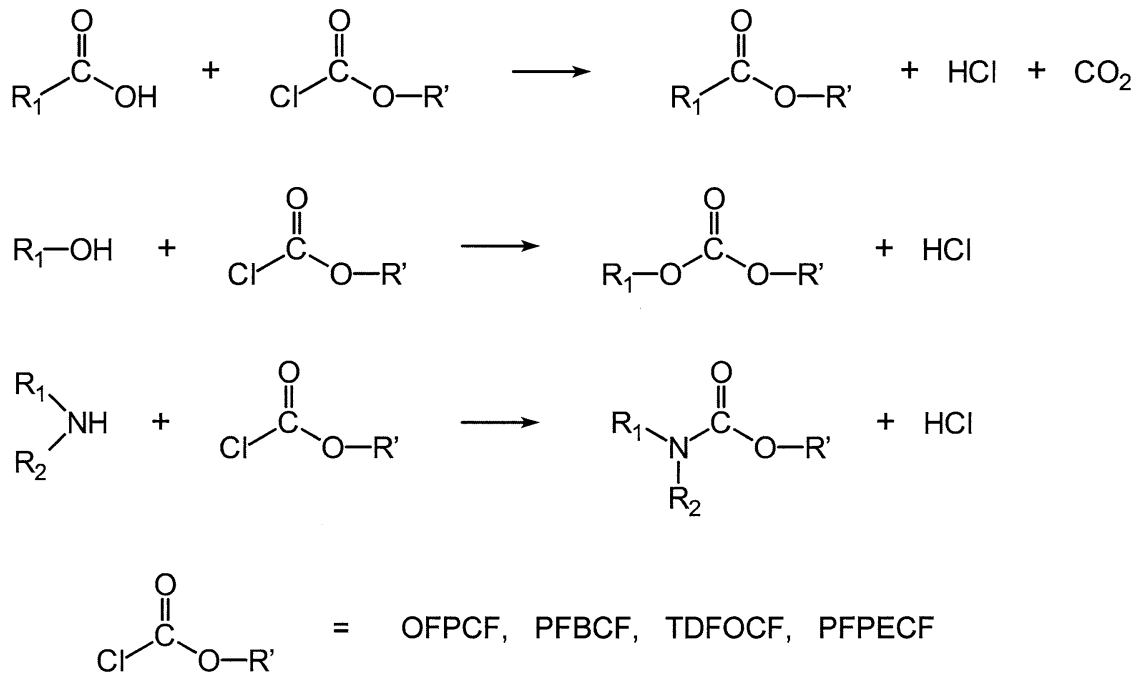

Scheme 2

on six candidate analytes representing five different classes $^{\circ}$ of $^{\circ}$ compounds $^{\circ}\left(\right.$ Table $\left.^{\circ} 1\right) .{ }^{\circ} \mathrm{OFPCF}^{\circ}$ and $^{\circ} \mathrm{PFBCF}$ were successful in derivatizing all the analytes, whereas TDFOCF failed with both 3-aminopropanol and valine, and PFPECF failed with 3-aminopropanol, valine, and 2-aminoethanol'(Table 1 ). The \%ower reactivityobserved for TDFOCF and PFPECF is probably attributable to the reduced electron-withdrawing effect exerted by the perfluorinated moiety on the chloroformate group.

From ${ }^{\circ}$ Table $^{\circ} 1$, $^{\text {it }}{ }^{\circ}$ is $^{\circ}$ evident $^{\circ}$ that ${ }^{\circ} \mathrm{OFPCF}^{\circ}$ accomplishes the complete substitution of all active hydrogens, whereas PFBCF introduces only one substituent on the amine groups of valine, 2-aminoethanol and 3aminopropanol, and two substituents on hydroxylamine. This was confirmed by PCI mass spectra. The lower degree of hydrogen substitution does not represent intrinsic evidence that PFBCF is less reactive than OFPCF toward the amine group, but indicates that the derivatization stops after the first substitution, as previously ${ }^{\circ}$ observed ${ }^{\circ}$ by $^{\circ} \operatorname{Simpson}^{\circ}$ and $^{\circ}$ coworkers $^{\circ}[20,21]$.

In order to compare the reactivities of OFPCF and PFBCF further, a derivatization procedure for 2aminoethanol, 3-aminopropanol, malic acid, and valine was performed using both derivatizing agents at once. Product distributions showed that 2-aminoethanol and 3-aminopropanol yielded both mixed products and derivatives containing only PFB terminals, (2aminoethanol: OFP/PFB 37\%, PFB/PFB 63\%; 3aminopropanol: OFP/PFB 21\%, PFB/PFB 79\%). Malic acid yielded only the OFPCF derivative (OFP/OFP $100 \%$ ). Valine yielded multiple derivatives carrying all the combinations of the two terminal groups (OFP/OFP $12 \%$, OFP/PFB 61\%, PFB/PFB 27\%). These data confirmed a slight preference of OFPCF for the carboxylic group and PFBCF for the aminic group.

The GC retention times of the OFPCF derivatives fall in the $13-15^{\circ} \mathrm{min}^{\circ}$ range $^{\circ}\left(\mathrm{Table}^{\circ} 1\right)^{\circ}{ }^{\circ}$ corresponding to ${ }^{\circ}$ oven temperatures of 200 to $230{ }^{\circ} \mathrm{C}$. This indicates that all OFPCF derivatives are relatively volatile because of the presence of multiple highly-fluorinated substituents, despite the relatively high molecular weights. This volatility represents an ideal condition for wide applicability and nondiscriminating GC injection. PFBCF derivatives eluted at considerably higher retention times $^{\circ}\left(\right.$ Table $\left.^{\circ} 1\right)$, ${ }^{\circ}$ corresponding $^{\circ}$ to $^{\circ}$ oven ${ }^{\circ}$ temperatures ${ }^{\circ}$ of 250 to $290{ }^{\circ} \mathrm{C}$. PFPECF produced even less volatile derivatives (for example, the resorcinol disubstituted derivative eluted at $20.31 \mathrm{~min}$ ). This would restrict the applicability of PFPECF derivatization to the simplest analytes.

\section{Mass Spectra of Highly-Fluorinated Derivatives}

ECNI mass spectra of most investigated derivatives showed considerable complexity. Therefore, interpreting the observed fragmentation patterns is necessary in order to apply this method to the search and identification of new DBPs from ozonation water treatments.

Figure $^{\circ} 2^{\circ}$ illustrates ${ }^{\circ}$ the ${ }^{\circ}$ type $^{\circ}$ of $^{\circ} \mathrm{ECNI}^{\circ}$ mass $^{\circ}$ spectra obtained using the four different derivatizing agents. In general, distinctive spectral patterns were present and structural features of the analytes could be deduced from the mass spectra. However, almost all derivatives showed extensive fragmentation, with molecular ions often absent in the mass spectra. PCI-MS was occasionally utilized for determining the molecular ions (and consequently the degree of hydrogen substitution in the derivatives), even if the sensitivity of PCI-MS was considerably lower (about two orders of magnitude) than ECNI-MS.

Certain spectral features are common to most derivatives. Both carbonate and carbamate groups contain weak chemical bonds and fragment on either side of the carbonyl group. For example, $\alpha$-cleavage fragments $[\mathrm{M}-\mathrm{ROCO}]^{-}$are evident in the ECNI mass spectra of the resorcinol derivatives (at $m / z 367$ for OFPCF, $m / z 333$ for PFBCF, $m / z 499$ for TDFOCF, and $m / z 363$ for PFPECF, Figure $\left.^{\circ} 2\right) .{ }^{\circ}$ These $^{\circ}$ processes $^{\circ}$ occur $^{\circ}$ in ${ }^{\circ}$ both $^{\circ}$ ion ${ }^{\circ}$ polarities, 


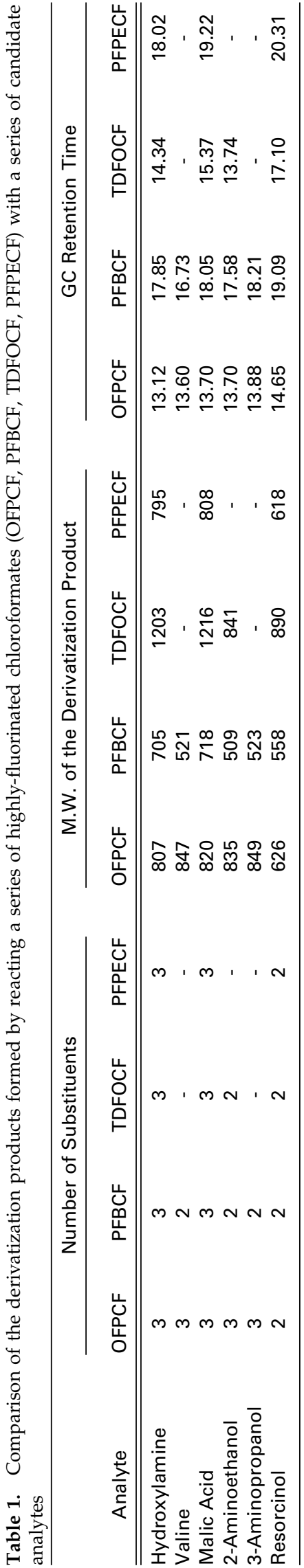

release the groups introduced in the derivatization, and may be followed by further fragmentation. For example, the OFPCF malic acid derivative consecutively releases one molecule of alcohol and one of $\mathrm{CO}_{2}(\mathrm{~m} / \mathrm{z}$ $544,{ }^{\circ}$ Figure $\left.^{\circ} 3 \mathrm{~b}\right) .^{\circ} \alpha$-Cleavage ${ }^{\circ}$ fragments $^{\circ}$ are $^{\circ}$ prevalent also in the PCI mass spectra of the derivatives, but the molecular weight information is more easily obtained. For example, the protonated molecular ion $(m / z 821)^{\circ}$ of $^{\circ}$ the ${ }^{\circ}$ malic $^{\circ}$ acid $^{\circ} \mathrm{OFPCF}^{\circ}$ derivative ${ }^{\circ}$ (Figure $3 a)^{\circ}$ consecutively ${ }^{\circ}$ release $^{\circ}{ }^{\text {neutral }}{ }^{\circ}$ molecules ${ }^{\circ}$ to $^{\circ}$ give fragments at $m / z 589(-\mathrm{ROH}), 545\left(-\mathrm{ROH}-\mathrm{CO}_{2}\right)$, and $313\left(-2 \mathrm{ROH}-\mathrm{CO}_{2}\right)$.

A slightly different fragmentation process is observed in the ECNI mass spectra of the aromatic analytes: in this case, a radical is released instead of a neutral molecule and the charge is sustained on the phenolic oxygen, yielding the ion $\left[\mathrm{M}-\mathrm{ROCO}^{-}\right.$as the most abundant of the spectrum. This behavior is observed for the PFBCF derivarives of 2,4-dihydroxybenzoic acid, 1,3,5-trihydroxybenzene, $m$ aminophenol, and the OFPCF derivatives of resorcinol and ${ }^{\circ} m$-aminophenol ${ }^{\circ}\left(\right.$ Table $\left.^{\circ} 2\right)$.

The relative intensity of the most significant ions in ECNI mass spectra of OFPCF and PFBCF derivatives are $^{\circ}$ reported $^{\circ}$ in $^{\circ}$ Table $^{\circ} 2 .^{\circ}$ Although ${ }^{\circ}$ the $^{\circ}$ origin $^{\circ}$ of ${ }^{\circ}$ these fragment ions is often not straightforward, a few considerations of general significance can be made.

1. The molecular ion is either missing or exhibits extremely low abundance.

2. Two analytes containing only carboxylic groups (methylmalonic and tricarballylic acids) exhibit rather unpredictable fragmentation patterns: cleavage of $\mathrm{C}-\mathrm{C}$ bonds occurs for the PFBCF derivative of methylmalonic acid $(\mathrm{m} / \mathrm{z} 253)$ and the OFPCF derivative of tricarballylic acid $(m / z 544)$, but not for the other two derivatives.

3. OFPCF derivatives of aliphatic hydroxycarboxylic acids (malic, tartronic, tartaric) typically release the alcoholic oxygen upon fragmentation ([M - $\mathrm{ROH}$ $\left.-\mathrm{CO}_{2}\right]^{-}$), while this cleavage does not take place in PFBCF derivatives.

4. Aromatic amine and phenol derivatives typically fragment by $\alpha$-clevage (the radical R-OCO is released, where $\mathrm{R}$ is either OFP- or PFB-). Subsequently a variety of consecutive dissociations take place (i.e., $\mathrm{RO}$, $\mathrm{CO}_{2}$, or $\mathrm{HF}$ are released). For example, the $m$ aminophenol OFP-derivative produces the ion at $\mathrm{m} / \mathrm{z}$ $624\left(\left[\mathrm{M}-\mathrm{ROCO}^{-}\right)\right.$as the base peak and the ion at $\mathrm{m} / \mathrm{z}$ $349\left(\left[\mathrm{M}-\mathrm{ROCO}-\mathrm{RO}-\mathrm{CO}_{2}\right]^{-}\right)$as the second most abundant in the ECNI mass spectrum. The corresponding PFB-derivative gives the primary fragment ion at $m / z 556\left(\left[\mathrm{M}-\mathrm{ROCO}^{-}\right)\right.$which subsequently releases a $\mathrm{CO}_{2}$ molecule $(\mathrm{m} / \mathrm{z}$ 512).

5. OFPCF derivatives of hydroxylamine, 2-aminoethanol, 3-aminopropanol, and valine all produce the ion at $\mathrm{m} / \mathrm{z} 532$ as the base peak in the ECNI mass spectrum. This peak can be attributed to the $\left[\mathrm{N}(\mathrm{COOR})_{2}\right]^{-}$ion, which is common for all the analytes, since double substitution on the amine group takes place in the 

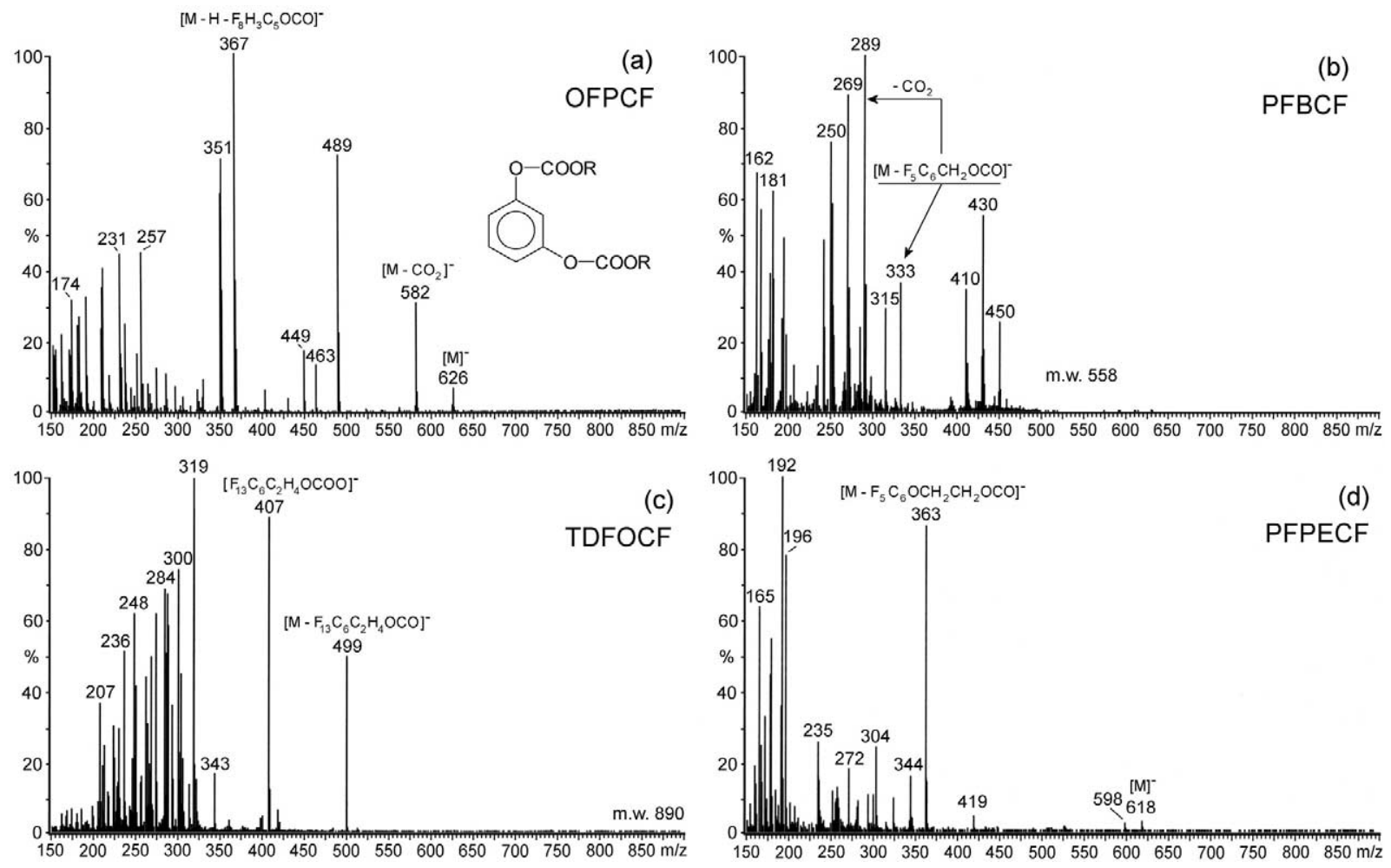

Figure 2. Electron capture negative ion mass spectra of resorcinol $(1 \mathrm{mg} / \mathrm{L})$ derivatized with: (a) OFPCF; (b) PFBCF; (c) TDFOCF; (d) PFPECF.

derivatization. This ion is highly indicative of the presence of an amine group in the analyte, but provides little information for other structural features of the derivative. In contrast, PFB-derivatives (with single substitution on the amine group) retains the charge in the analyte hydrocarbon backbone during ECNI fragmentation, which primarily involves $\alpha$-clevage.

6. Since $\alpha$-cleavage is generally the expected major dissociation process, $\alpha$-cleavage fragments, along
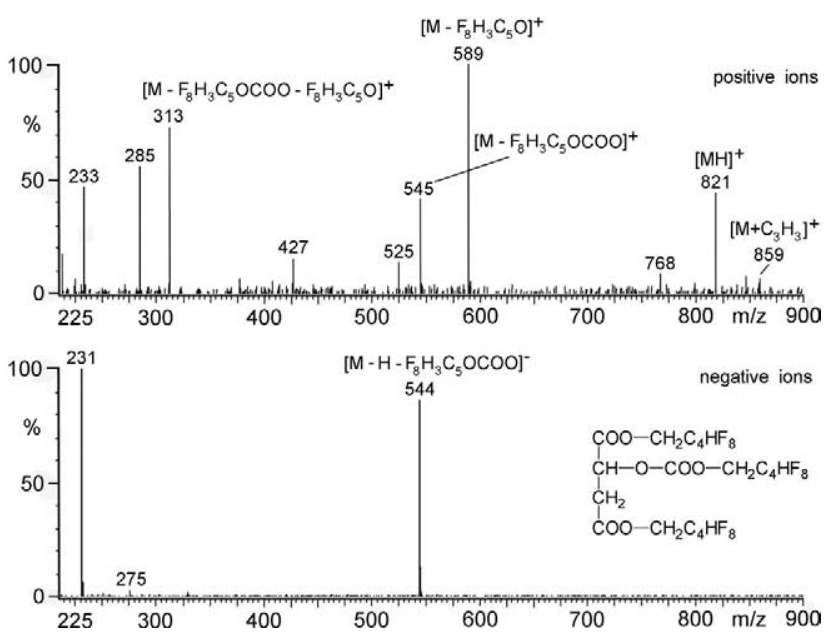

Figure 3. Positive and negative electron capture chemical ionization mass spectra of the malic acid $(1 \mathrm{mg} / \mathrm{L})$ OFPCF tri-derivative. with the knowledge on the number of substituted hydrogens, serve as good indicators of the derivative structure.

The ECNI mass spectrum of the 2-aminoethanol $\mathrm{TDFOCF}^{\circ}$ derivative, ${ }^{\circ}$ depicted ${ }^{\circ}{ }^{\circ}{ }^{\circ}$ Figure $^{\circ} 4$, ${ }^{\circ}$ represents a typical fragmentation pattern for TDFOCF derivatives, which, in general, are only scarcely informative. The higher $\mathrm{m} / \mathrm{z}$ ion $(\mathrm{m} / \mathrm{z} 431)$ corresponds to [M $-\mathrm{ROCO}-\mathrm{F}]^{-}$. Since a double radical loss is unlikely, the ion likely originates from the consecutive release of two (or three) neutral molecules, each requiring a hydrogen rearrangement, such as $\left[\mathrm{M}-(\mathrm{R}-\mathrm{H})-\mathrm{CO}_{2}\right.$ $-\mathrm{HF}]^{-}$. The rest of the mass spectrum is unclear: a large number of peaks is evident in the $m / z 200-320$ range, most of which can be attributed to the fragmentation of the perfluorocarbon groups introduced by derivatization.

\section{Analytical Performance}

From the previous discussion, it appears that TD FOCF and PFPECF failed to react with some of the analytes tested. Moreover, their derivatives exhibit high GC retention times and scarcely informative ECNI mass spectra. Therefore, the subsequent quality assurance program was restricted to OFPCF and PFBCF. The overall analytical performance of the method was tested using the extended set of target 


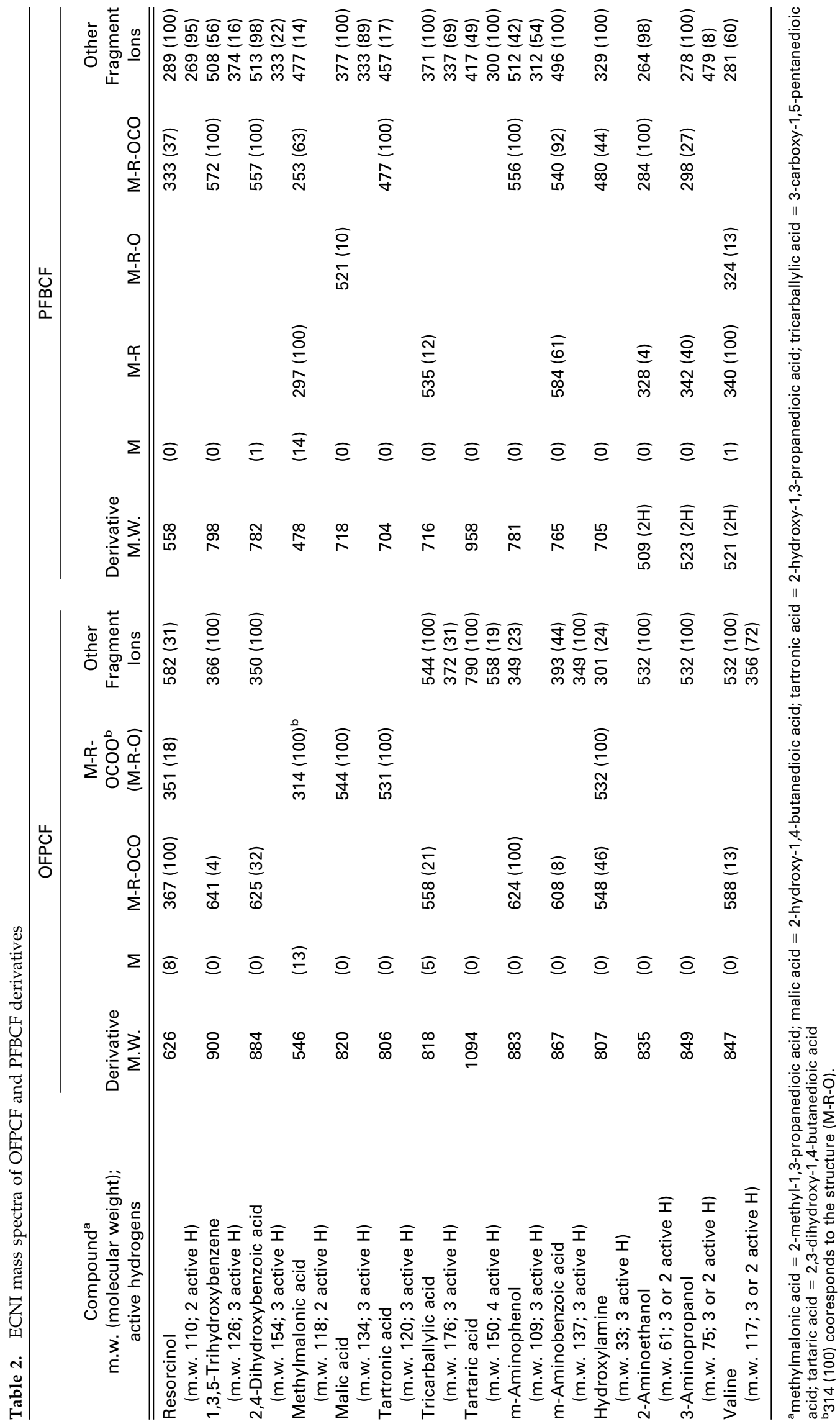




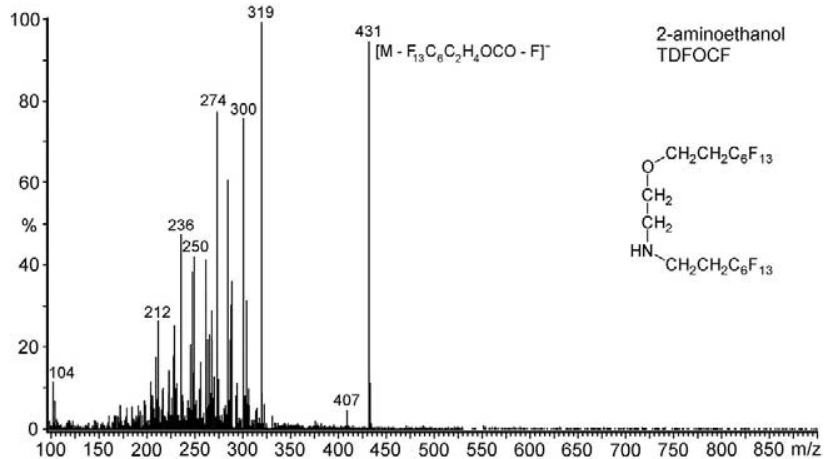

Figure 4. Electron capture negative ion mass spectrum of the 2-aminoethanol (1 mg/L) TDFOCF bi-derivative.

analytes $^{\circ}$ listed $^{\circ}$ in $^{\circ}$ Table $^{\circ} 2 .^{\circ}$ The $^{\circ}$ following ${ }^{\circ}$ analytical features were investigated: (1) repeatability of the derivatization; (2) repeatability of the instrumental response; (3) detection limits for the various analytes; (4) linearity of the calibration curves. These results, summarized ${ }^{\circ}$ in $^{\circ}$ Table $^{\circ} 3,^{\circ}$ refer $^{\circ}$ to $^{\circ}$ the $^{\circ}$ quantitative determination of target analytes.

The instrumental repeatability was tested by injecting five times each day the same derivatization mixtures arising from $100 \mu \mathrm{g} / \mathrm{L}$ aqueous analyte solutions, over five consecutive days. The standard deviations, expressed as percentage of the mean signal, ranged from 3 to $12 \%$, demonstrating good stability of the derivatization products and acceptable reproducibility of the ECNI-MS conditions. Further confirmation of product ion stability was obtained by analyzing the same hexane solutions at regular time intervals over a month (stored at $4{ }^{\circ} \mathrm{C}$ between analyses).

The repeatability of the derivatization process was measured by treating five aliquots of $100 \mu \mathrm{g} / \mathrm{L}$ analyte solutions using the protocol described in the experi-

Table 3. Analytical features for the derivatizations $(2 \mathrm{~mL})$ performed with OFPCF and PFBCF, including (i) the percentage standard deviation for repeated analysis of the same sample (instrumental repeatability); (ii) the percentage standard deviation for repeated derivatization of the same analytical solution (derivatization repeatability); (iii) the observed detection limit (S/N > 5), expressed as the analyte concentration in the starting water solution; (iv) the linearity range of the calibration curve; (v) the angular coefficient ( \pm standard deviation) and (vi) the square correlation factor for the bilogarithmic calibration curves. All experiments were performed in the ECNI-MS-SIM mode, by selecting the ions listed in Table 2 within appropriate retention time windows

\begin{tabular}{|c|c|c|c|c|c|c|}
\hline & \multicolumn{6}{|c|}{ OFPCF } \\
\hline & $\begin{array}{c}\text { Instr.Rep. } \\
(\sigma \%)\end{array}$ & $\begin{array}{c}\text { Der.Rep. } \\
(\sigma \%)\end{array}$ & $\begin{array}{l}\text { Det.Lim. } \\
(\mu \mathrm{g} / \mathrm{L})\end{array}$ & $\begin{array}{c}\text { Cal.Range } \\
(\mu \mathrm{g} / \mathrm{L})\end{array}$ & $\begin{array}{c}\log / \log \\
\text { Ang.Coeff. }\end{array}$ & $r^{2}$ \\
\hline Resorcinol & 3.0 & 5.5 & 0.1 & $0.3-300$ & $1.01 \pm 0.02$ & 0.998 \\
\hline 1,3,5-Trihydroxybenzene & 7.8 & 8.3 & 0.3 & $0.3-500$ & $1.18 \pm 0.11$ & 0.998 \\
\hline 2,4-Dihydroxybenzoic acid & 4.4 & 8.1 & 0.3 & $0.3-500$ & $0.94 \pm 0.02$ & 0.997 \\
\hline Methylmalonic acid & 5.4 & 7.4 & 3 & $5-500$ & $1.00 \pm 0.03$ & 0.990 \\
\hline Malic acid & 6.2 & 9.8 & 0.3 & $0.3-300$ & $0.86 \pm 0.02$ & 0.992 \\
\hline Tartronic acid & 3.7 & 7.4 & 0.3 & $1-500$ & $0.89 \pm 0.09$ & 0.992 \\
\hline Tricarballylic acid & 6.8 & 8.9 & 1 & $1-500$ & $0.97 \pm 0.08$ & 0.980 \\
\hline Tartaric acid & 8.5 & 11.7 & 0.3 & $0.3-500$ & $0.86 \pm 0.02$ & 0.992 \\
\hline m-Aminophenol & 5.7 & 19.1 & 1 & $1-500$ & $0.98 \pm 0.02$ & 0.996 \\
\hline m-Aminobenzoic acid & 7.1 & 15.3 & 5 & $5-500$ & $1.15 \pm 0.04$ & 0.989 \\
\hline Hydroxylamine & 6.3 & 13.9 & 0.3 & $0.3-300$ & $0.85 \pm 0.02$ & 0.994 \\
\hline 2-Aminoethanol & 11.7 & 25.0 & 10 & $10-300$ & $1.23 \pm 0.12$ & 0.979 \\
\hline 3-Aminopropanol & 10.6 & 22.1 & 10 & $10-300$ & $1.26 \pm 0.11$ & 0.975 \\
\hline \multirow[t]{3}{*}{ Valine } & 8.3 & 19.4 & 10 & $10-500$ & $1.21 \pm 0.04$ & 0.982 \\
\hline & \multicolumn{6}{|c|}{ PFBCF } \\
\hline & $\begin{array}{l}\text { Instr.Rep. } \\
\qquad(\sigma \%)\end{array}$ & $\begin{array}{l}\text { Der.Rep. } \\
(\sigma \%)\end{array}$ & $\begin{array}{l}\text { Det.Lim. } \\
(\mu \mathrm{g} / \mathrm{L})\end{array}$ & $\begin{array}{c}\text { Cal.Range } \\
(\mu \mathrm{g} / \mathrm{L})\end{array}$ & $\begin{array}{c}\log / \log \\
\text { Ang.Coeff. }\end{array}$ & $r^{2}$ \\
\hline Resorcinol & 5.2 & 14.3 & 0.1 & $0.3-100$ & $0.70 \pm 0.09$ & 0.986 \\
\hline $1,3,5$-Trihydroxybenzene & 10.1 & 21.1 & 100 & $100-500$ & $0.98 \pm 0.07$ & 0.980 \\
\hline 2,4-Dihydroxybenzoic acid & 9.0 & 16.6 & 30 & $30-500$ & $1.28 \pm 0.12$ & 0.943 \\
\hline Methylmalonic acid & 5.6 & 24.3 & 100 & $100-500$ & $0.98 \pm 0.09$ & 0.970 \\
\hline Malic acid & 6.8 & 25.6 & 10 & $10-500$ & $1.08 \pm 0.04$ & 0.986 \\
\hline Tartronic acid & 4.1 & 18.6 & 1 & $1-500$ & $0.92 \pm 0.05$ & 0.969 \\
\hline Tricarballylic acid & 7.5 & 21.6 & 5 & $5-500$ & $1.11 \pm 0.10$ & 0.982 \\
\hline Tartaric acid & 7.6 & 29.4 & 100 & $100-500$ & $1.22 \pm 0.12$ & 0.966 \\
\hline m-Aminophenol & 9.9 & 12.1 & 30 & $100-500$ & $1.31 \pm 0.15$ & 0.953 \\
\hline m-Aminobenzoic acid & 7.1 & 9.6 & 30 & $100-500$ & $1.23 \pm 0.14$ & 0.989 \\
\hline Hydroxylamine & 5.4 & 11.4 & 30 & $100-500$ & $0.75 \pm 0.14$ & 0.980 \\
\hline 2-Aminoethanol & 10.4 & 10.3 & 3 & $5-500$ & $1.11 \pm 0.05$ & 0.983 \\
\hline 3-Aminopropanol & 11.0 & 12.4 & 1 & $1-100$ & $0.92 \pm 0.09$ & 0.972 \\
\hline Valine & 9.3 & 15.3 & 10 & $10-500$ & $1.03 \pm 0.06$ & 0.982 \\
\hline
\end{tabular}


mental section. An internal standard (perfluorotriphenylphosphine) was added to each sample before GC-MS analysis. Standard deviations ranging from 5 to $12 \%$ were observed for polycarboxylic acids, polyhydroxycarboxylic acids, and polyhydroxybenzenes derivatized with OFPCF. Derivatization of analytes with an amine group exhibited lower repeatability $(14 \%<\sigma \%$ $<25 \%$ ). For derivatizations performed with PFPCF, the opposite was observed: variability was lower for aminoalcohols and aminoacids $(10 \%<\sigma \%<15 \%)$ than for hydroxy-carboxylic compounds $(14 \%<\sigma \%<30 \%)$. These findings, combined with the results from competitive derivatization experiments (where OFPCF and PFBCF were mixed) suggest that the repeatability is correlated with the reaction kinetics. In fact, it appears that the faster the derivatization takes place, the more repeatable quantitative determinations are.

Detection limits and calibration ranges proved excellent for the derivatizations performed with OFPCF, with absolute detection limits in the low fmol range for hydroxycarboxylic acids, di- and trihydroxybenzenes, and $^{\circ}$ hydroxylamine ${ }^{\circ}\left(\right.$ Figure $\left.^{\circ} 5\right) .{ }^{\circ}$ For $^{\circ}$ these ${ }^{\circ}$ compounds, further concentration of the final hexane solutions can yield detection limits in the tens of ppt (ng/L) range. Calibration curves were calculated in bilogarithmic scales, so that homogeneous distribution of data points
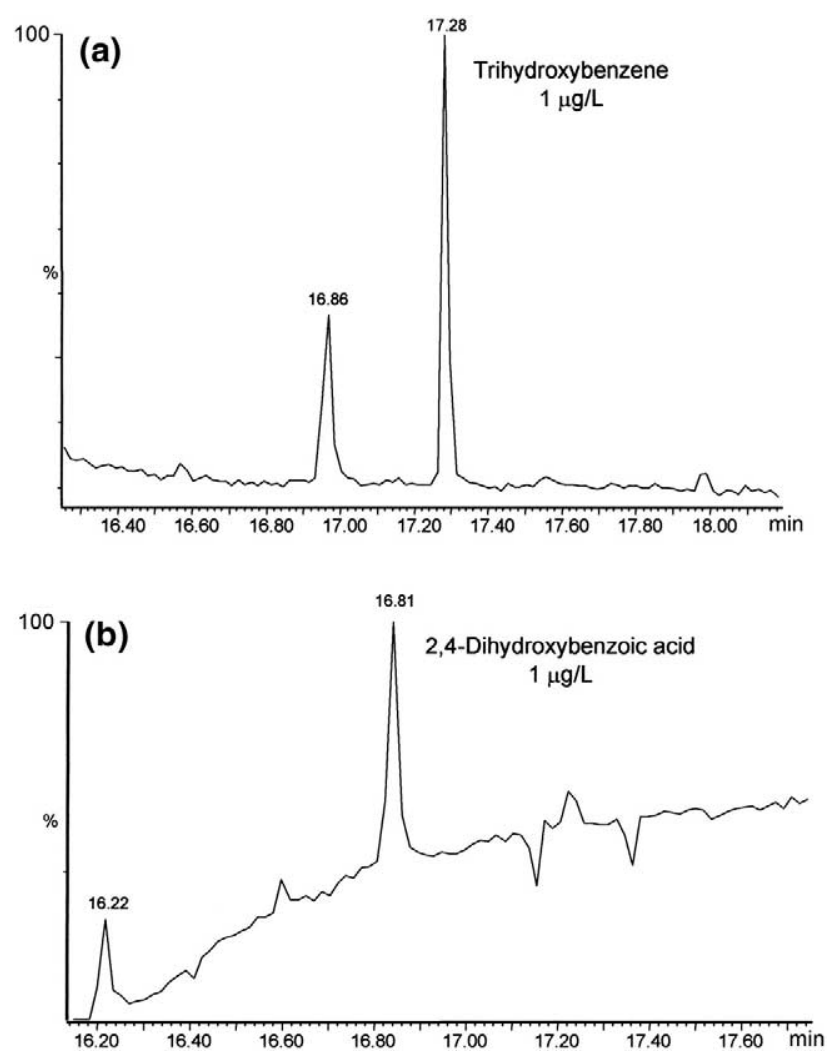

Figure 5. Selected ion mass chromatograms obtained from OFPCF derivatization of a $1 \mu \mathrm{g} / \mathrm{L}$ solution of (a) 1,3,5trihydroxybenzene and (b) 2,4-dihydroxybenzoic acid. Selected ions are $\mathrm{m} / \mathrm{z} 641$ and 366 for 1,3,5-trihydroxybenzene and $\mathrm{m} / \mathrm{z}$ 625 and 350 for 2,4-dihydroxybenzoic acid. along the calibration range was obtained. Linear plots with satisfactory correlation factors were observed $\left(\mathrm{r}^{2}=\right.$ 0.975 to 0.998$)$.

Considerably higher detection limits were observed for the derivatizations performed with PFBCF (typically hundreds of fmol), with the remarkable exception of aminoalcohols, which could be determined in the low fmol range. Also, the calibration curves for PFBCF derivatives exhibited more limited linearity ranges, with slopes deviating from unity and lower correlation factors than for OFPCF $\left(\mathrm{r}^{2}>0.943\right.$ for PFBCF derivatives; $r^{2}>0.975$ for OFPCF derivatives). It can be concluded that OFPCF is a better performing derivatizing agent than PFBCF for most polar analytes, and its use is recommended for survey analyses of unknown analytes, such as the search for unidentified drinking water DBPs.

Further tests to validate our derivatization protocol were made to evaluate possible effects of an actual drinking water matrix. Since our effort focused on the detection of drinking water DBPs, comparative experiments were performed, using tap water instead of distilled water, to prepare standard and analytical solutions ${ }^{\circ}$ of $^{\circ}$ the ${ }^{\circ}$ candidate $^{\circ}$ analytes ${ }^{\circ}$ listed $^{\circ}$ in $^{\circ}$ Table $^{\circ} 2 .{ }^{\circ}$ No differences were observed, and the overall variability of the experimental data matched that determined for distilled ${ }^{\circ}$ water $^{\circ}$ solutions $^{\circ}\left(\right.$ Table $\left.^{\circ} 3\right) .{ }^{\circ} \mathrm{We}^{\circ}$ also $^{\circ}$ wanted $^{\circ}$ to make sure that the presence of multiple polar components did not suppress their derivatization. Therefore, the derivatization procedure with OFPCF and PFBCF was performed on a mixture of eleven candidate analytes. The GC profile obtained from one of these experiments $^{\circ}$ is ${ }^{\circ}$ reported $^{\circ}$ in $^{\circ}$ Figure $^{\circ} 6,^{\circ}$ and $^{\circ}$ shows $^{\circ}$ that $^{\circ}$ the OFPCF derivatization occurred with the usual efficiency for all the analytes. Similar results were also observed for PFBCF derivatization.

\section{Application to the Detection of Ozonation By-Products}

In order to verify the derivatization procedure in an application of real interest, water solutions of Suwannee River Fulvic Acid (SRFA) were subjected to oxidative treatment with ozone for variable time intervals. The resulting solutions were subsequently derivatized with OFPCF, and the final hexane solutions were analyzed by GC-MS, under full-scan conditions, in order to identify any possible SRFA oxidation products. Blank samples of untreated SRFA solutions were derivatized and analyzed, to rule out the components already present in SRFA and the derivatization by-products.

From this comparative analysis, three SRFA ozonation by-products were identified, namely malic acid, tricarballylic acid, and 1,2,3-benzenetricarboxylic acid. To our knowledge, these have not been previously reported as ozonation by-products. A previous study did report two other benzenetricarboxylic acid DBPs in ozonated drinking water: the 1,2,4- and 1,3,5-isomers 


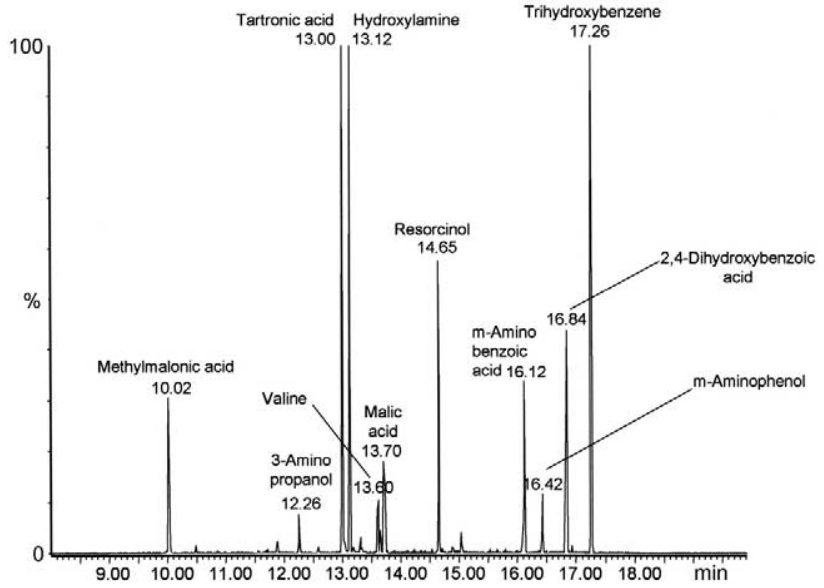

Figure 6. Selected ion mass chromatogram obtained from OFPCF derivatization of a eleven standard analyte solutions (500 $\mu \mathrm{g} / \mathrm{L}$ each). Signals from all the selected ions are added together.

[23]. While ${ }^{\circ}$ determining ${ }^{\text {the }}$ first 9 two acids 9 presented no difficulty as the mass spectra of their derivatives were already known, attributing the mass spectrum of the third component as 1,2,3-benzenetricarboxylic acid required mass spectral interpretation and subsequent confirmation with a pure standard. This ECNI spectrum presents a weak molecular ion at $\mathrm{m} / \mathrm{z} 852(1 \%)$, primary fragment ions at $\mathrm{m} / \mathrm{z} 652\left(2 \%,\left[\mathrm{M}-\mathrm{C}_{4} \mathrm{~F}_{8}\right]^{-}\right), \mathrm{m} / \mathrm{z}$ $\left.635\left(7 \% \text {, [M - }-\mathrm{H}-\mathrm{CHF}_{2}\left(\mathrm{CF}_{2}\right)_{3} \mathrm{CH}_{2}\right]^{-}\right), \mathrm{m} / \mathrm{z} 231(87 \%$, $\left.\left[\mathrm{CHF}_{2}\left(\mathrm{CF}_{2}\right)_{3} \mathrm{CH}_{2} \mathrm{O}\right]^{-}\right)$, and a main secondary fragment ion at $m / z 403\left(100 \%,\left[635-\mathrm{CHF}_{2}\left(\mathrm{CF}_{2}\right)_{3} \mathrm{CH}_{2} \mathrm{OH}\right]^{-}\right)$. In the positive ion mode, the DBPs were not present at high enough concentrations to enable full PCI mass spectra to be obtained, but SIM profiles matched those of the pure standards. Systematic investigation of ozonation by-products from humic and fulvic acid solutions, as well as from real drinking water from treatment plants, is underway.

\section{Conclusions}

Highly-fluorinated chloroformates proved to be powerful derivatizing agents for polar hydrophilic analytes, which react directly in aqueous solution, avoiding the risk of incomplete extraction and recovery of the polar analytes. Four different fluorinated chloroformates could be easily synthesized and all of them proved capable of carrying out direct aqueous sample derivatization. However, among them, OFPCF exhibited superior performance in terms of general applicability, sensitivity, and linearity of response, while PFBCF found specific application in the analysis of aminoalcohols. While most derivatizing agents undergo rapid decomposition in water, the insolubility of highlyfluorinated chloroformates considerably slows down their hydrolysis kinetics.

A unique feature of OFPCF and PFBCF derivatizations is their effectiveness in reacting with carboxylic, hydroxylic, and aminic groups at once, releasing multiply-substituted derivatives that can be easily extracted and determined by GC-(ECNI)-MS. The entire procedure from raw aqueous sample to ready-to-inject hexane solution of the derivatives requires less than ten $\mathrm{min}$. Another positive aspect of this procedure is that it produces stable derivatives, with optimal volatility for GC separation, and high electron-affinity, which allows their detection as negative ions at trace level.

We believe that the easy preparation of these derivatizing agents, combined with their wide applicability and unique analytical features, open up innovative and still unexplored avenues in several application fields, including clinical, environmental, and food chemistry. Promising results are expected in the determination of unknown water disinfection by-products for which only the hydrophobic and moderately hydrophilic fractions have been adequately characterized. While these fractions are prevalent in chlorinated drinking water, ozone is expected to produce a large variety of highly polar by-products, which remain to be identified. We are hopeful that the use of direct methods, such as the OFPCF and PFBCF derivatizations presented here, will enable their identification.

\section{Acknowledgments}

The authors gratefully acknowledge financial support from the U.S. Environmental Protection Agency (U.S.-EPA; Cooperative Agreement no. R-82795101-1), M.I.U.R, and Regione Piemonte. They also thank Dr. Patrizia Davit for assistance with ozonation reactions. This paper has been reviewed in accordance with the U.S. EPA's peer and administrative review policies and approved for publication. Mention of trade names or commercial products does not constitute endorsement or recommendation for use by the U.S. EPA.

\section{References}

1. Weinberg, H. S. Disinfection By-Products in Drinking Water: The Analytical Challenge. Anal. Chem. 1999, 71, 801A-808A.

2. Richardson, S. D. Drinking Water Disinfection By-Products. In The Encyclopedia of Environmental Analysis and Remediation, Vol. III; Meyers R. A., Ed.; John Wiley and Sons: New York, 1998, pp 1398-1421.

3. Glaze, W. H.; Weinberg H. S. Identification and Occurrence of Ozonation By-Products in Drinking Water; American Water Works Association Research Foundation: Denver, CO, 1993.

4. Pelizzetti, E.; Maurino, V.; Minero, C.; Vincenti, M. Disinfection By-Products in Drinking-Water Treatments. Mechanism of Formation, Analysis, and Research Needs. Chim. Ind. (Rome) 1994 76, 701-707.

5. Plewa, M. J.; Wagner, E. D.; Jazwierska, P.; Richardson, S. D.; Chen, P. H.; McKague, A. B. Halonitromethane Drinking Water Disinfection By-Products: Chemical Characterization and Mammalian Cell Cytotoxicity and Genotoxicity. Environ. Sci. Technol. 2004, 38, 62-68.

6. Richardson, S. D.; Caughran, T. V.; Poiger, T.; Guo, Y.; Crumley, F. G. Application of DNPH Derivatization with LC/MS to the Identification of Polar Carbonyl Disinfection By-Products in Drinking Water. Ozone Sci. Eng. 2000, 22, 653-675.

7. Zwiener, C.; Glauner, T.; Frimmel, F. H. Method Optimization for the Determination of Carbonyl Compounds in Disinfected 
Water by DNPH Derivatization and LC-ESI-MS-MS. Anal. Bioanal. Chem. 2002, 372, 615-621.

8. Richardson, S. D.; Karst, U. A New Tailor-Made Derivatizing Agent for Identifying Polar Carbonyl DBPs in Drinking Water. Proceedings of the 221st American Chemical Society National Meeting; San Diego, CA, April, 2001.

9. Identification of New and Uncharacterized Disinfection By-Products in Drinking Water; International Life Sciences Institute: Washington, D.C. 1999.

10. Hušek, P. Chloroformates in Gas Chromatography as General Purpose Derivatizing Agents. J. Chromatogr. B 1998, 717, 57-91.

11. Minero, C.; Vincenti, M.; Pelizzetti, E. Determination of Ethylene Glycol in Aqueous Matrix by Direct Derivatization with Hexyl Chloroformate. Ann. Chim. (Rome) 1993, 83, 511-521.

12. Minero, C.; Vincenti, M.; Lago, S.; Pelizzetti, E. Determination of Trace Amounts of Highly Hydrophilic Compounds in Water by Direct Derivatization and Gas ChromatographyMass Spectrometry. Fresenius J. Anal. Chem. 1994, 350, 403-409.

13. Vincenti, M.; Minero, C.; Lago, S.; Rovida, C. Determination of Hydroxycarbamates in Aqueous Matrices by Direct Derivatization and GC-MS Analysis in Chemical Ionization Mode. J. High Res. Chromatogr. 1995, 18, 359-362.

14. Angelino, S.; Maurino, V.; Minero, C.; Pelizzetti, E.; Vincenti, M. Improved Procedure for $n$-Hexyl Chloroformate-Mediated Derivatization of Highly Hydrophilic Substances Directly in Water: Hydroxyaminic Compounds. J. Chromatogr. A 1998, 793, 307-316.

15. Hall, B. J.; Parikh, A. R.; Brodbelt, J. S. Aqueous Phase Hexylchloroformate Derivatization and Solid Phase Microextraction: Determination of Benzoylecgonine in Urine by Gas Chromatography-Quadrupole Ion Trap Mass Spectrometry. J. Forensic Sci. 1999, 44, 527-534.

16. Maurino, V.; Minero, C.; Pelizzetti, E.; Angelino, S.; Vincenti, M. Ultratrace Determination of Highly Hydrophilic Compounds by 2,2,3,3,4,4,5,5,-Octafluoropentyl Chloroformate-Mediated Derivatization Directly in Water. J. Am. Soc. Mass Spectrom. 1999, 10, 1328-1336.

17. Vincenti, M.; Ghiglione, N.; Valsania M. C.; Davit, P.; Richardson, S. D. Synthesis of Highly Fluorinated Chloroformates and Their Use as Derivatizing Agents for Hydrophilic Compounds and Drinking-Water-Disinfection By-Products. Helv. Chim. Acta 2004, $87,370-375$

18. Abe, I.; Fujimoto, N.; Nishiyama, T.; Terada, K.; Nakahara, T. Rapid Analysis of Amino Aacid Enantiomers by Chiral-Phase Capillary Gas Chromatography. J. Chromatogr. A 1996, 722, 221-227.

19. Lee, J. M.; Norton, S. J. Pyrethroid-Like Carbamates Having Insecticidal Activity. J. Agric. Food Chem. 1990, 38, 273-278.

20. Simpson, J. T.; Torok, D. S.; Markey, S. P. Pentafluorobenzyl Chloroformate Derivatization for Enhancement of Detection of Amino Acids or Alcohols by Electron Capture Negative Ion Chemical Ionization Mass Spectrometry. J. Am. Soc. Mass Spectrom. 1995, 6, 525-528.

21. Simpson, J. T.; Torok, D. S.; Girard, J. E.; Markey, S. P. Analysis of Amino Acids in Biological Fluids by Pentafluorobenzyl Chloroformate Derivatization and Detection by Electron Capture Negative Ionization Mass Spectrometry. Anal. Biochem. 1996, 233, 58-66.

22. Gordon, G.; Cooper, W. J.; Rice, R. G.; Pacey, G. E. Disinfectant Residual Measurement Methods, 2nd ed.; American Water Works Association Research Foundation: Denver, CO, 1992, 327-332.

23. Richardson, S. D.; Thruston, A. D., Jr.; Caughran, T. V.; Chen, P. H.; Collette, T. W.; Floyd, T. L.; Schenck, K. M.; Lykins, B. W., Jr.; Sun, G.-R.; Majetich, G. Identification of New Ozone Disinfection By-Products in Drinking Water. Environ. Sci. Technol. 1999, 33, 3368-3377. 